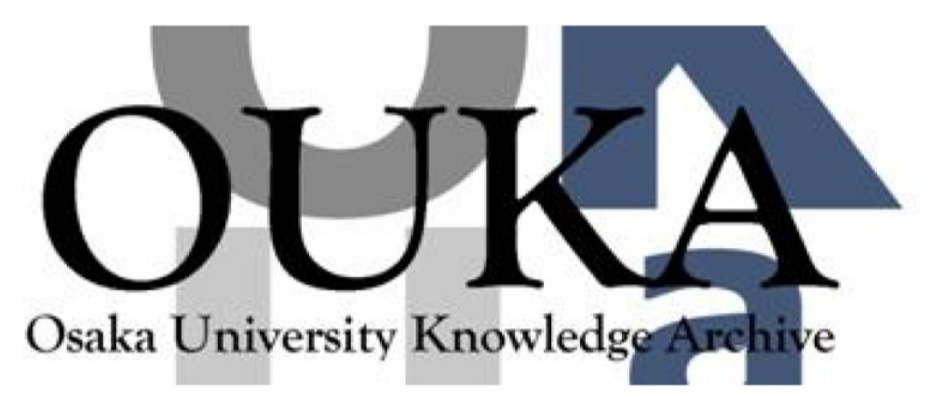

\begin{tabular}{|c|l|}
\hline Title & $\begin{array}{l}\text { Shallow and diffuse spin-orbit potential for } \\
\text { proton elastic scattering from neutron-rich } \\
\text { helium isotopes at } 71 \mathrm{MeV} / \text { nucleon }\end{array}$ \\
\hline Author(s) & Sakaguchi, S.; Uesaka, T.; Aoi, N. et al. \\
\hline Citation & $\begin{array}{l}\text { Physical Review C - Nuclear Physics. 87(2) } \\
\text { p. } 21601-p .21601\end{array}$ \\
\hline Issue Date & $2013-02-20$ \\
\hline oaire:version & VoR \\
\hline URL & https://hdl. handle. net/11094/51207 \\
\hline rights & o2013 American Physical Society \\
\hline Note & \\
\hline
\end{tabular}

Osaka University Knowledge Archive : OUKA

https://ir. Library. osaka-u. ac. jp/

Osaka University 


\title{
Shallow and diffuse spin-orbit potential for proton elastic scattering from neutron-rich helium isotopes at $71 \mathrm{MeV} /$ nucleon
}

\author{
S. Sakaguchi, ${ }^{1,}{ }^{*}$ T. Uesaka, ${ }^{2}$ N. Aoi, ${ }^{2}$ Y. Ichikawa, ${ }^{3}$ K. Itoh, ${ }^{4}$ M. Itoh,${ }^{5}$ T. Kawabata,,${ }^{1}$ T. Kawahara, ${ }^{6}$ Y. Kondo,${ }^{7}$ H. Kuboki,${ }^{3}$ \\ T. Nakamura, ${ }^{7}$ T. Nakao, ${ }^{3}$ Y. Nakayama, ${ }^{7}$ H. Sakai, ${ }^{3}$ Y. Sasamoto, ${ }^{1}$ K. Sekiguchi, ${ }^{2}$ T. Shimamura, ${ }^{7}$ Y. Shimizu, ${ }^{1}$ and T. Wakui ${ }^{5}$ \\ ${ }^{1}$ Center for Nuclear Study, University of Tokyo, Tokyo 113-0001, Japan \\ ${ }^{2}$ RIKEN Nishina Center, Saitama 351-0198, Japan \\ ${ }^{3}$ Department of Physics, University of Tokyo, Tokyo 113-0033, Japan \\ ${ }^{4}$ Department of Physics, Saitama University, Saitama 338-8570, Japan \\ ${ }^{5}$ Cyclotron \& Radioisotope Center, Tohoku University, Miyagi 980-8578, Japan \\ ${ }^{6}$ Department of Physics, Toho University, Chiba, Japan \\ ${ }^{7}$ Department of Physics, Tokyo Institute of Technology, Tokyo 152-8551, Japan
}

(Received 22 November 2012; published 20 February 2013)

\begin{abstract}
Vector analyzing powers for proton elastic scattering from ${ }^{8} \mathrm{He}$ at $71 \mathrm{MeV} /$ nucleon have been measured using a solid polarized proton target operated in a low magnetic field of $0.1 \mathrm{~T}$. The spin-orbit potential obtained from a phenomenological optical model analysis is found to be significantly shallower and more diffuse than the global systematics of stable nuclei, which is an indication that the spin-orbit potential is modified for scattering involving neutron-rich nuclei. A close similarity between the matter radius and the root-mean-square radius of the spin-orbit potential is also identified.
\end{abstract}

DOI: 10.1103/PhysRevC.87.021601

PACS number(s): 25.60.Bx, 24.70.+s, 25.40.Cm, 29.25.Pj

The strong spin-orbit coupling in atomic nuclei plays an important role in nuclear structure and reactions. One good example is the spin-orbit splitting of single-particle levels, which is a key ingredient for the success of the nuclear shell model [1,2]. Spin-orbit coupling is also responsible for many other phenomena such as the dominance of the prolate shape and the emergence of the isomeric intruder state. Moreover, in terms of nuclear reactions, spin-orbit coupling is responsible for the polarization effects in elastic scattering. There has recently been renewed interest in spin-orbit coupling since it is predicted to be modified in neutron-rich nuclei. A number of experimental results suggest a change in the shell structure of neutron-rich nuclei that could be explained by a reduction in the spin-orbit splitting [3-5]. However, there has been no experimental study examining how the spin-orbit coupling is modified in nuclear reactions of unstable nuclei.

Spin asymmetry in proton-nucleus $(p-A)$ elastic scattering is a prominent manifestation of the spin-orbit coupling in nuclear reactions. The coupling is generally represented by a spin-orbit term in the optical model potential, i.e., the spinorbit potential. Current understanding of this potential has been based on extensive measurements and analysis of the vector analyzing powers for elastic scattering of polarized protons from various stable nuclei over a wide energy range [6-11]. It is now well established that the shape and magnitude of the spin-orbit potential does not depend strongly on the target nucleus. The shape is reasonably expressed by a derivative of the density distribution [12-14], while the magnitude is almost independent of the mass number [10,11]. However, whether these systematics hold even in regions far from the stability line is still an open question. The structure of

*Present address: Department of Physics, Kyushu University, Fukuoka 812-8581, Japan; sakaguchi@phys.kyushu-u.ac.jp neutron-rich nuclei often shows distinctive features such as a very diffuse nuclear surface, a neutron skin and halo, and a difference between the radial dependence of the proton and neutron distributions. From the surface nature of the spin-orbit coupling, we can expect that the spin-orbit potential is modified in the neutron-rich region. In this Rapid Communication we determine the spin-orbit potential between a proton and a typical neutron-rich nucleus ${ }^{8} \mathrm{He}$ and investigate the effect of the exotic structure of the neutron-rich nucleus on the spin-orbit coupling in $p$ - $A$ scattering.

Determination of the spin-orbit potential requires vector analyzing power data, and until several years ago, such data could not be obtained in the experiment with a radioactiveion beam. This was due to the lack of polarized targets that can be operated at a low magnetic field of $\ll 1 \mathrm{~T}$. However, we were able to construct a solid polarized proton target at $0.1 \mathrm{~T}$ based on a new polarizing method [15-18] and have applied it to scattering experiments of ${ }^{6} \mathrm{He}$ at $71 \mathrm{MeV} /$ nucleon $[17,19,20]$.

Recently, we measured the vector analyzing powers for proton elastic scattering from ${ }^{8} \mathrm{He}$ at $71 \mathrm{MeV} /$ nucleon. These neutron-rich helium isotopes are suitable for exploring the modification of spin-orbit potential, since they have large neutron-excess ratios $(N-Z) / A$ and significantly diffuse density distributions. The data were analyzed with a phenomenological optical model to discuss the overall characteristics of the spin-orbit interaction with a least-biased approach. Details of both the measurements and analysis are reported in this paper.

The analyzing power measurement of $p-{ }^{8} \mathrm{He}$ elastic scattering was carried out at RI Beam Factory operated by RIKEN Nishina Center and Center for Nuclear Study, University of Tokyo. The ${ }^{8} \mathrm{He}$ beam was produced by a projectile fragmentation reaction of an ${ }^{18} \mathrm{O}$ beam with an energy of $100 \mathrm{MeV} /$ nucleon bombarding a 13-mm-thick Be target. The 
${ }^{8} \mathrm{He}$ particles were then separated by the RIKEN Projectilefragment Separator (RIPS) [21]. The energy of the ${ }^{8} \mathrm{He}$ beam was $71.0 \pm 1.4 \mathrm{MeV} /$ nucleon at the center of the secondary target. The typical intensity and purity of the beam were $1.5 \times 10^{5} \mathrm{pps}$ and $77 \%$, respectively. As a secondary target, the solid polarized proton target [15-18] was placed at the final focal plane of the RIPS. The target was operated under a low magnetic field of $91 \mathrm{mT}$, which allowed us to detect low-energy $(\sim 10 \mathrm{MeV})$ recoil protons under inverse kinematics conditions. The average target polarization was $11.3 \pm 2.6 \%$.

The detector system is the same as that used in the $p-{ }^{6} \mathrm{He}$ measurement described in Ref. [20] except for the recoil proton detectors. To achieve higher angular and energy resolutions for the recoil protons, we used multi-wire drift chambers (MWDCs) and CsI(Tl) scintillators with a Si PIN photodiode readout. The position resolution of the MWDCs was $200 \mu \mathrm{m}$ (full width at half maximum). This corresponds to an angular resolution of $0.05^{\circ}$ in sigma in the center-of-mass system, which is one order of magnitude better than that in the $p-{ }^{6} \mathrm{He}$ measurement. The effects of the magnetic field on the proton scattering angle, which was comparable to or smaller than the detector resolution, were properly corrected in the data analysis. Using the correlation between the recoil and scattered particle scattering angles, a clear peak corresponding to the $p-{ }^{8} \mathrm{He}$ elastic scattering was identified. Spurious asymmetries such as imbalances in the detector efficiency and solid angle were canceled out by reversing the direction of target polarization. It should be emphasized again that the operation of the polarized target in a low magnetic field allowed us to detect recoil protons with an angular resolution sufficient to identify the elastic scattering events.

The measured differential cross sections $(d \sigma / d \Omega)$ and analyzing powers $\left(A_{y}\right)$ for $p-{ }^{8} \mathrm{He}$ (present) and $p-{ }^{6} \mathrm{He}[19,20]$ are shown in Fig. 1 as filled circles and squares, respectively. Published $d \sigma / d \Omega$ data [22] are also plotted as the open symbols. It is known from extensive measurements at $65 \mathrm{MeV}$ [9] that the analyzing powers for $p-A$ scattering from stable nuclei usually take large positive values of $\sim 0.9$ at the second peak, except for the $p-{ }^{4} \mathrm{He}$ case in which $A_{y}$ is almost zero [23]. The present $A_{y}$ data for $p-{ }^{8} \mathrm{He}$ and $p-{ }^{6} \mathrm{He}$ lie between these two cases.

To determine the spin-orbit potentials between a proton and ${ }^{8} \mathrm{He}$ nucleus, we perform a phenomenological optical model analysis. For the optical model potential, we use a Woods-Saxon form factor with a Thomas-type spin-orbit

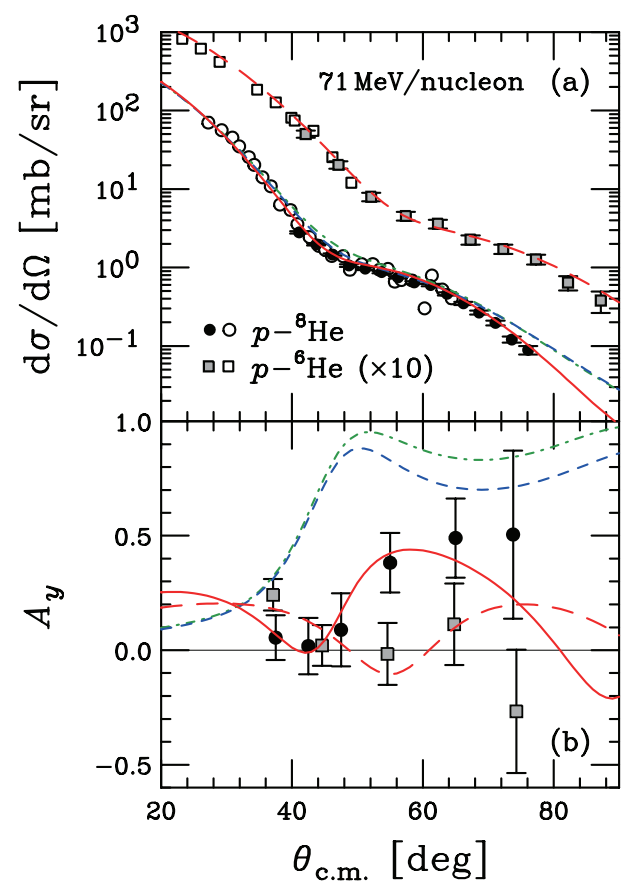

FIG. 1. (Color online) Differential cross section (upper) and analyzing power (lower) of $p^{-6,8} \mathrm{He}$ elastic scattering at $71 \mathrm{MeV} /$ nucleon.

term:

$$
\begin{aligned}
U_{\mathrm{OM}}(R)= & -V_{0} f_{r}(R)-i W_{0} f_{i}(R) \\
& +V_{s} \frac{2}{R} \frac{d}{d R} f_{s}(R) \boldsymbol{L} \cdot \boldsymbol{\sigma}_{p}+V_{\mathrm{C}}(R),
\end{aligned}
$$

with

$$
\begin{aligned}
f_{x}(R) & =\left[1+\exp \left(\frac{R-r_{0 x} A^{1 / 3}}{a_{x}}\right)\right]^{-1} \\
(x & =r, i, \text { or } s) .
\end{aligned}
$$

Here, $\boldsymbol{R}$ is the relative coordinate between a proton and a ${ }^{8} \mathrm{He}$ particle, $R$ represents $|\boldsymbol{R}|, \boldsymbol{L}=\boldsymbol{R} \times\left(-i \hbar \nabla_{R}\right)$ is the associated angular momentum, and $\sigma_{p}$ is the Pauli spin operator of the proton. The subscripts $r, i$, and $s$ denote the real and imaginary parts of the central term and the real part of the spin-orbit term, respectively. $V_{0}, W_{0}$, and $V_{s}$ are depth parameters of the corresponding terms. $r_{0 x}$ and $a_{x}$ are radius and diffuseness parameters, respectively. $V_{\mathrm{C}}$ is a Coulomb potential of uniformly charged sphere with a radius of $r_{0 \mathrm{C}} A^{1 / 3}$ $\mathrm{fm}\left(r_{0 \mathrm{C}}=1.3 \mathrm{fm}\right)$. No surface absorption term is considered

TABLE I. Parameters of the optical potentials for $p-{ }^{6} \mathrm{Li}$ at $72 \mathrm{MeV} /$ nucleon [25], $p-{ }^{6} \mathrm{He}$ at $71 \mathrm{MeV} /$ nucleon [20], and $p-{ }^{8} \mathrm{He}$ at $71 \mathrm{MeV} /$ nucleon (present work).

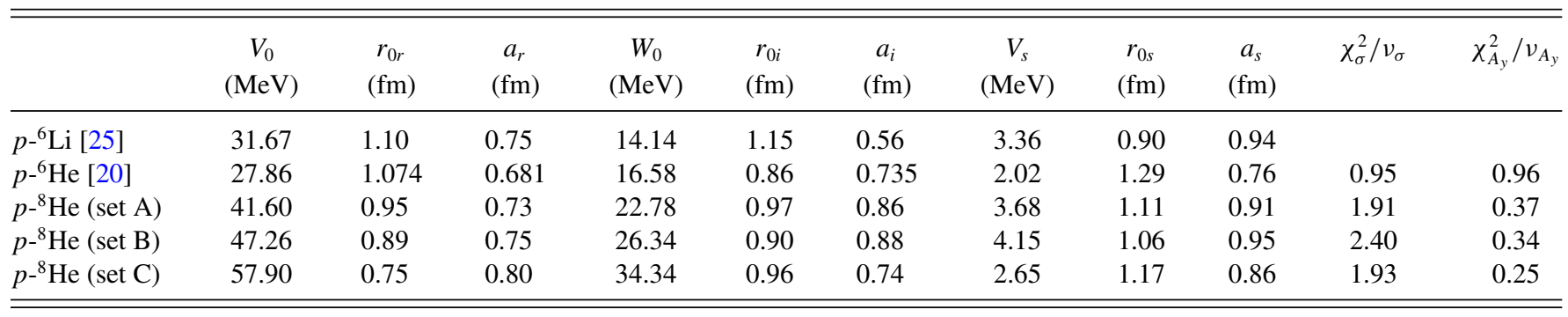


here. Since the statistics is limited, the imaginary part of the spin-orbit potential is not included in the fits. If we assume it is as small as in the case of stable nuclei, the effect on $A_{y}$ is within the error bars. However, because it is still unknown whether this assumption holds in unstable nuclei, the imaginary spin-orbit potential should be investigated in the future when sufficient data are available.

Using the optical potential given in Eqs. (1) and (2), we search for a parameter set that reproduces both the $d \sigma / d \Omega$ and $A_{y}$ data obtained in the present work and the $d \sigma / d \Omega$ data of Ref. [22]. The fit is carried out using the ECIS79 code [24]. The initial values are taken from a set of parameters for $p-{ }^{6} \mathrm{Li}$ elastic scattering at $72 \mathrm{MeV} /$ nucleon [25]. The solid and long-dashed curves in Fig. 1 show the best-fit results for $p-{ }^{8} \mathrm{He}$ and $p-{ }^{6} \mathrm{He}$, respectively. The reduced chi-square values for $d \sigma / d \Omega$ and $A_{y}$ are minimized as $\chi_{\sigma}^{2} / \nu_{\sigma}=1.91$ and $\chi_{A_{y}}^{2} / \nu_{A_{y}}=0.37$, respectively, in the $p-{ }^{8} \mathrm{He}$ case. The optical potential parameters of $p-{ }^{6} \mathrm{Li}[25]$ and $p-{ }^{6} \mathrm{He}$ [20] and those obtained for $p{ }^{8} \mathrm{He}$ (set A) are summarized in Table I. These three potentials are similar to each other, probably because of the resemblance of density distribution. Since ${ }^{6} \mathrm{Li}$ is also a weakly bound nucleus, its matter radius and $d \sigma / d \Omega$ are almost identical with those for ${ }^{6} \mathrm{He}$ as described in Ref. [20]. However, we should note that it is not straightforward to deal with the spin-orbit potential for the ${ }^{6} \mathrm{Li}$ case, because it has a nonzero spin. Henceforth, the quantitative discussion focuses on the nuclei with spin zero.

The upper panel of Fig. 2 presents the radial dependence of the central terms of the $p{ }^{8} \mathrm{He}$ potential (set A). The solid, dashed, and dot-dashed curves denote the present potential, that obtained by Koning and Delaroche (KD03) [11], and that obtained by Varner et al. (CH89) [10], respectively. A surface absorption term is included in the imaginary term in the case of the global potentials. While the ${ }^{8} \mathrm{He}$ nucleus is located outside the applicable range of these two global potentials, they serve as guides for comparison since their mass-number dependence is not strong, especially for the spin-orbit term. The real and imaginary terms of the present potential are in reasonable agreement with the global potentials. The root-mean-square (rms) radii and volume integral of each term are summarized in Table II. The real and imaginary terms of the present potential are comparable to those of the global potentials.

The lower panel of Fig. 2 displays the radial dependence of $R V_{l s}(R)$, which is defined as

$$
R V_{l s}(R)=2 V_{s} \frac{d}{d R}\left[1+\exp \left(\frac{R-r_{0 s} A^{1 / 3}}{a_{s}}\right)\right]^{-1}
$$

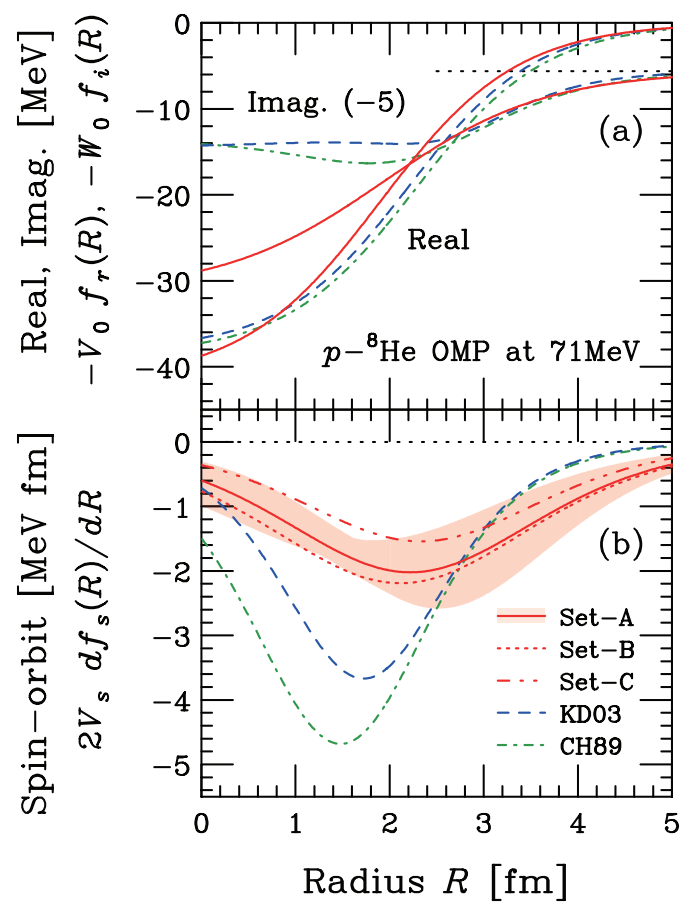

FIG. 2. (Color online) Radial dependence of the optical potential between a proton and a ${ }^{8} \mathrm{He}$ nucleus.

Here, the $R$ factor on the left-hand side is introduced to cancel the $1 / R$ term of the Thomas function in order to present the shape of potential without divergence at small radii. The solid line in Fig. 2 (lower) shows the best-fit potential (set A) with a statistical error band (shaded area) corresponding to a potential with $\Delta \chi_{A_{y}}^{2} \equiv \chi_{A_{y}}^{2}-\chi_{A_{y} \text { :min. }}^{2}=1$. To check the fitting ambiguity of the spin-orbit potential, we search for other possible parameter sets. Excluding very unusual potentials such as ones with $V_{0}>60 \mathrm{MeV}$, ten different sets are obtained. In Table I, two of them are presented: sets B and C are the results with the deepest and the shallowest spin-orbit potentials, respectively. They are approximately consistent with that of set A within the statistical error band as shown in the lower panel of Fig. 2. The obtained spin-orbit potentials have broad peaks at $R \sim 2.2 \mathrm{fm}$, whereas the global potentials (dashed and dot-dashed) have sharper peaks at smaller radii of $R \sim 1.6 \mathrm{fm}$. The spin-orbit potential for ${ }^{8} \mathrm{He}$ is found to be shallower and more diffuse than the global systematics of stable nuclei.

TABLE II. Volume integral and rms radius of each term of the $p^{-6,8} \mathrm{He}$ potentials at $71 \mathrm{MeV} / \mathrm{nucleon}$.

\begin{tabular}{|c|c|c|c|c|c|c|c|}
\hline & & \multicolumn{3}{|c|}{$\left(\mathrm{MeV} \mathrm{fm}^{3}\right)$} & \multicolumn{3}{|c|}{$(\mathrm{fm})$} \\
\hline \multirow{2}{*}{${ }^{6} \mathrm{He}$} & Ref. [20] & 320 & 144 & $66_{-26}^{+24}$ & 2.95 & 2.98 & $3.33_{-0.26}^{+0.23}$ \\
\hline & CH89 & 466 & 232 & 108 & 3.01 & 3.25 & 2.29 \\
\hline \multirow[b]{2}{*}{${ }^{8} \mathrm{He}$} & set A & 371 & 261 & $107_{-41}^{+35}$ & 3.08 & 3.52 & $3.58_{-0.20}^{+0.25}$ \\
\hline & KD03 & 413 & 191 & 95 & 3.04 & 3.22 & 2.52 \\
\hline
\end{tabular}




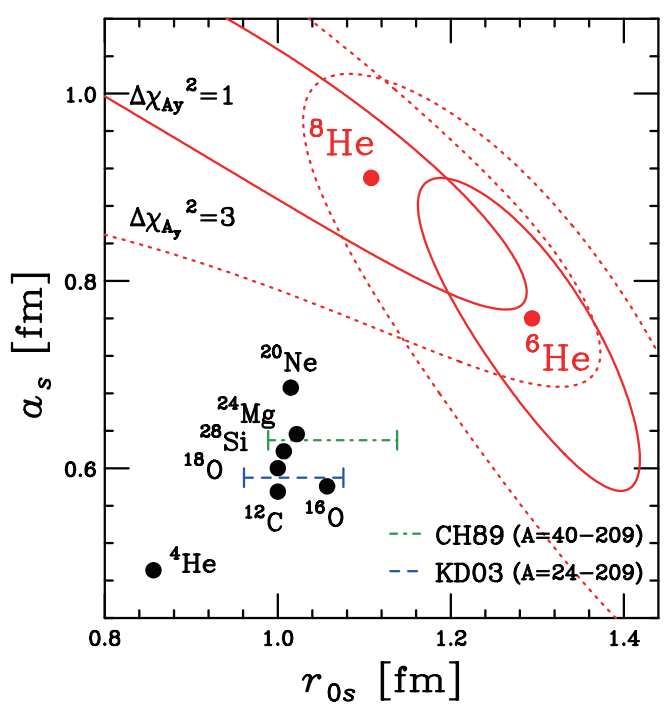

FIG. 3. (Color online) Two-dimensional distribution of the radius and diffuseness parameters of the spin-orbit term of the local (filled circles) and global (dot-dashed: CH89, dashed: KD03) potentials for spin-zero nuclei. Solid and dotted lines indicate $\Delta \chi_{A_{y}}^{2}=1$ and 3, respectively.

To examine the effect of spin-orbit potential on the observables, we compare the results of calculations using different spin-orbit potentials but with identical central potentials. The short-dashed and dot-dashed lines in Fig. 1 correspond to the results of calculations using the same central terms as the present potential but with the spin-orbit terms of the KD03 and CH89 potentials, respectively. These "standard" spin-orbit potentials give large positive $A_{y}$ values that are incompatible with the current data. It should be stressed that the shallow and diffuse spin-orbit potential is essential in reproducing the present $A_{y}$ data.

In Fig. 3, the parameters $r_{0 s}$ and $a_{s}$ are presented for comparison. Filled circles show the parameters determined for spin-zero nuclei ranging from ${ }^{4} \mathrm{He}$ to ${ }^{28} \mathrm{Si}[9,20,26]$. Parameters for heavier nuclei are represented by the global potentials, KD03 (dashed) and CH89 (dot-dashed), which overlap those of the light nuclei. The present results (set A for ${ }^{8} \mathrm{He}$ ) are shown by the filled red circles with uncertainties evaluated in the following manner: For each point in the $r_{0 s}-a_{s}$ plane, a depth parameter $V_{s}$ is re-searched to minimize the $\chi_{A_{y}}^{2}$ value. The solid and dotted lines in the figure indicate regions where $\Delta \chi_{A_{y}}^{2}=1$ and 3 , respectively. The radius and diffuseness parameters of the spin-orbit potentials obtained for the neutron-rich helium isotopes appear to be larger than those for the stable nuclei. In contrast, the depth parameters for ${ }^{6} \mathrm{He}$ and ${ }^{8} \mathrm{He}$, determined as $2.02_{-0.86}^{+0.82}$ and $3.68_{-0.91}^{+0.80} \mathrm{MeV}$, respectively, are smaller than the typical value of $\sim 5 \mathrm{MeV}$.

The shape and magnitude of the spin-orbit potential can be discussed in terms of the rms radius $\left\langle r_{l s}^{2}\right\rangle^{1 / 2}$ and the amplitude of $R V_{l s}(R)$ at the peak position. These quantities provide more robust features of the spin-orbit potentials than the individual parameters that couple with each other. Figure 4(a) shows the mass-number dependence of the $\left\langle r_{l s}^{2}\right\rangle^{1 / 2}$ values of the potentials for the spin-zero nuclei. The symbols are

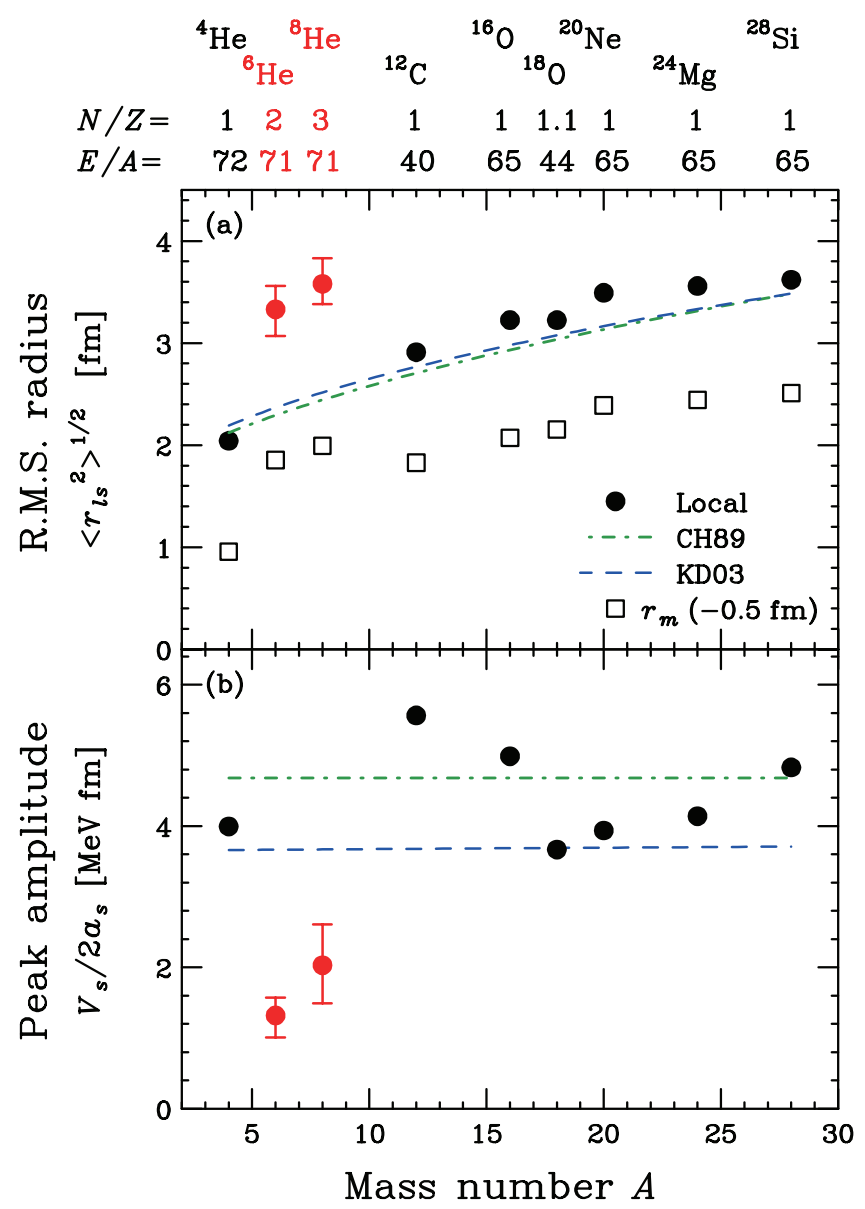

FIG. 4. (Color online) The $\left\langle r_{l s}^{2}\right\rangle^{1 / 2}$ and $r_{m}$ (upper) values and the peak amplitude $V_{s} / 2 a_{s}$ (lower) of the spin-orbit potentials for light spin-zero nuclei. For ${ }^{8} \mathrm{He}$, the results of set $\mathrm{A}$ are shown. Those with sets $\mathrm{B}$ and $\mathrm{C}$ are consistent with them within the statistical uncertainties. The symbols for $r_{m}$ are shifted horizontally by $-0.5 \mathrm{fm}$ to prevent overlap.

the same as those in Fig. 3. We can see that the $\left\langle r_{l s}^{2}\right\rangle^{1 / 2}$ values of the present potentials (in red; $3.33_{-0.26}^{+0.23} \mathrm{fm}$ for ${ }^{6} \mathrm{He}$ and $3.58_{-0.20}^{+0.25} \mathrm{fm}$ for ${ }^{8} \mathrm{He}$ ) are significantly larger than the systematics of the stable nuclei. Moreover, it is interesting to note that a close similarity is found between the mass-number dependence of $\left\langle r_{l s}^{2}\right\rangle^{1 / 2}$ and that of the matter radius $r_{m}$ [27-30], plotted as the open squares in Fig. 4(a). The enhancement seen in the $r_{m}$ values of ${ }^{6} \mathrm{He}$ and ${ }^{8} \mathrm{He}$ is more distinct in the behavior of the $\left\langle r_{l s}^{2}\right\rangle^{1 / 2}$ values, which indicates the particular sensitivity of the spin-orbit interaction to the nuclear surface structure.

Figure 4(b) displays the amplitude of $R V_{l s}(R)$ at the peak position $R=r_{0 s} A^{1 / 3}$, which is denoted by $V_{s} / 2 a_{s}$. The peak amplitudes of the local potentials for a stable nuclei are in the range $3.5-5.5 \mathrm{MeV} f \mathrm{fm}$ and are almost independent of the mass number. Those of the global potentials (dashed and dot-dashed lines) are consistent with these amplitudes. However, the peak depths of the present potentials, $1.32_{-0.21}^{+0.25} \mathrm{MeV}$ fm for ${ }^{6} \mathrm{He}$ and $2.03_{-0.54}^{+0.58} \mathrm{MeV}$ fm for ${ }^{8} \mathrm{He}$, are smaller than the standard values. From these results, we can conclude that the spin-orbit potentials between a proton and neutron-rich ${ }^{6} \mathrm{He}$ and ${ }^{8} \mathrm{He}$ 
nuclei are both shallower and more diffuse than the global systematics of nuclei along the stability line. This is considered to be a consequence of the diffuse density distribution of these neutron-rich isotopes.

In summary, vector analyzing powers have been measured for the proton elastic scattering from ${ }^{8} \mathrm{He}$ at $71 \mathrm{MeV} /$ nucleon to investigate the spin-orbit potential between a proton and a neutron-rich ${ }^{8} \mathrm{He}$ nucleus. The measured differential cross sections and analyzing powers were analyzed using a phenomenological optical model to derive the overall characteristics of the $p{ }^{6,8} \mathrm{He}$ interactions. The spin-orbit potentials for ${ }^{6} \mathrm{He}$ and ${ }^{8} \mathrm{He}$ were found to be both shallower and more diffuse than the global systematics of stable nuclei. The rms radius of these spin-orbit potentials deviate from the well-established mass-number dependence and show a close similarity to the behavior of the matter radius. Depths of the obtained potentials were found to be significantly reduced from the standard value. The shallow and diffuse spin-orbit potentials for ${ }^{6} \mathrm{He}$ and ${ }^{8} \mathrm{He}$ are considered to be a consequence of the diffuse density distribution of these two neutron-rich helium isotopes.

The authors thank the staff at the RIKEN Nishina Center and CNS for operating the accelerators and ion source during the measurement. We acknowledge Y. Iseri, M. Tanifuji, and S. Ishikawa for fruitful discussions. This work was supported by MEXT KAKENHI Grant No. 17684005. S.S. acknowledges financial support from JSPS KAKENHI Grant No. 18-11398.
[1] M. G. Mayer, Phys. Rev. 75, 1969 (1949).

[2] O. Haxel, J. J. D. Jenson, and H. Suess, Phys. Rev. 75, 1766 (1949).

[3] J. Dobaczewski, I. Hamamoto, W. Nazarewicz, and J. A. Sheikh, Phys. Rev. Lett. 72, 981 (1994).

[4] G. A. Lalazissis, D. Vretenar, W. Pöschl, and P. Ring, Phys. Lett. B 418, 7 (1998).

[5] T. Otsuka, T. Matsuo, and D. Abe, Phys. Rev. Lett. 97, 162501 (2006).

[6] R. Craig et al., Nucl. Phys. 58, 515 (1964).

[7] L. N. Blumberg et al., Phys. Rev. 147, 812 (1966).

[8] V. Comparat et al., Nucl. Phys. A 221, 403 (1974).

[9] H. Sakaguchi et al., Phys. Rev. C 26, 944 (1982).

[10] R. L. Varner et al., Phys. Rep. 201, 58 (1991).

[11] A. J. Koning and J. P. Delaroche, Nucl. Phys. A 713, 231 (2003).

[12] C. B. Dover and N. Van Giai, Nucl. Phys. A 190, 373 (1972).

[13] R. R. Scheerbaum, Nucl. Phys. A 257, 77 (1976).

[14] E. Bauge, J. P. Delaroche, and M. Girod, Phys. Rev. C 58, 1118 (1998).

[15] T. Uesaka et al., Nucl. Instrum. Methods Phys. Res., Sect. A 526, 186 (2004)
[16] T. Wakui et al., Nucl. Instrum. Methods Phys. Res., Sect. A 550, 521 (2005).

[17] M. Hatano et al., Eur. Phys. J. A 25, 255 (2005).

[18] T. Wakui, in Proceedings of the XIth International Workshop on Polarized Ion Sources and Polarized Gas Targets 2005, edited by T. Uesaka, H. Sakai, A. Yoshimi, and K. Asahi (World Scientific, Singapore, 2007).

[19] T. Uesaka et al., Phys. Rev. C 82, 021602(R) (2010).

[20] S. Sakaguchi et al., Phys. Rev. C 84, 024604 (2011).

[21] T. Kubo et al., Nucl. Instrum. Methods Phys. Res., Sect. B 70, 309 (1992).

[22] A. A. Korsheninnikov et al., Nucl. Phys. A 617, 45 (1997).

[23] S. Burzynski et al., Phys. Rev. C 39, 56 (1989).

[24] J. Raynal, ECIS code, CEA-R2511 Report, 1965 (unpublished).

[25] R. Henneck et al., Nucl. Phys. A 571, 541 (1994).

[26] E. Fabrici et al., Phys. Rev. C 21, 830 (1980).

[27] I. Angeli, At. Data Nucl. Data Tables 87, 185 (2004).

[28] I. Tanihata et al., Phys. Lett. B 289, 261 (1992).

[29] G. D. Alkhazov, A. V. Dobrovolsky, and A. A. Lobodenko, Nucl. Phys. A 734, 361 (2004).

[30] O. Kiselev et al., Eur. Phys. J. A 25, 215 (2005). 